\title{
HapticPulse - Reveal your Heart Rate in Physical Activities
}

\author{
Janko Timmermann ${ }^{1}$, Benjamin Poppinga ${ }^{1}$, Susanne Boll ${ }^{2}$, Wilko Heuten ${ }^{1}$ \\ 1 OFFIS - Institute for Information Technology, Germany \\ firstname.lastname@offis.de \\ http://www.offis.de/ \\ 2 University of Oldenburg, Germany \\ boll@informatik. uni-oldenburg. de \\ http://medien.informatik. uni-oldenburg.de/
}

\begin{abstract}
The heart rate is an objective parameter indicating the current physical activity. Displaying it to the user will help her or him to gain awareness of the physical load during certain activities. Current systems do not use the sense of touch to display the actual heart rate. Using the sense of touch has been shown to be potentially less distracting than using other senses in certain situations. In this paper we describe a system which displays the heart rate of the user using the sense of touch. We conducted a user study in the field with ten participants to collect qualitative and quantitative data, which serves as a guideline for the future improvement of such systems.
\end{abstract}

\section{Introduction}

The heart rate is one of the main parameters representing the level of physical activity. To know the current heart rate is essential for appropriate physical exercises. For example, it is important to keep a specific heart rate range regarding to your training goals [1][10] or even to increase the heart rate variability [2][5] which is a potential marker for health [8]. Measuring the heart rate is very common during sport activities [1].

Experienced runners or cyclists are usually aware of their physical load, their approximate heart rate, and what ranges are suitable for certain exercises. However, people that are new to physical activities have difficulties to understand or feel the necessary load and are often overloaded or underloaded. Such people are for example patients, who are required to reach a particular physical load over a certain time, in order to improve their cardiovascular system after a heart disease. Overstraining can cause further heart problems, while understraining does not have any effect on the improvement of the cardiovascular system. Another example of a user group are people with obesity who need to increase their physical effort. However, in order to keep the motivation of training in the long-term perspective, it is necessary to guide them from the beginning, in particular starting with small exercises, which do not overburden the user. Besides subjective measurements, e.g. asking people how they feel, the heart rate is an objective 
parameter indicating the current physical activity. Displaying the heart rate to the user will help her or him to gain awareness of physical load during certain activities and enables the user to adjust her or his physical effort according to the recommendations from doctors or training goals.

While many stationary home exercise machines have integrated sensors and displays, there are also watches measuring and displaying the heart rate with the help of a chest belt, which can be used during nearly any outdoor activity. The display usually consists of a number, i.e. the presentation of heartbeats in a minute. Users need to look at the display on demand to perceive this number and to interpret its meaning. Some of these watches also support notification mechanisms with tactile or auditory feedback to alert the user when specified heart rates are exceeded or deceeded.

However, none of these systems are using touch to display the current and actual heart rate, although there has been a lot of research indicating the beneficial use of touch. For example, the sense of touch can handle abstract messages [4] and has shown to be less distracting in certain situations [3][7]. Using it to communicate information to the user enables her or him to get the desired information without using other senses like the visual one by looking at a graphical display. In addition, tactile displays can be perceived without any further interaction of the user. If designed appropriately, they enable users to gain a continuous awareness of the presented information - in our case the heart rate. Furthermore, users can immediately feel the impact of increasing or decreasing the physical load in relation to the heart rate.

In this paper we describe a preliminary system that displays the user's heart rate using the sense of touch. We conducted a field study with 10 users to collect qualitative and quantitative data to serve as a guideline for the future improvement of this system or development of similar systems. The study showed, that users can actually learn about their heart rate by using the HapticPulse and correctly feel it with a certain accuracy. We found out that the device strengthened the awareness of the heart rate. Besides this, we collected interesting qualitative feedback for future improvements for such systems.

\section{Related Work}

Several approaches are existing that use displaying the heartbeat to create intimacy between two people. Lotan et al. (2007) propose a device "[...] for augmenting intimate or meditative moments between people at a distance [...]". The device has an outline of hands on the surface where the users can place their hands. It is then able to reflect the users' heartbeat or even to simultaneously use two vibration motors to show the heartbeat of the local user and a user of another device [6]. Werner et al. (2008) created a similar approach in which two partners wear rings which enable the wearer to feel the partner's heartbeat with the help of a small vibration motor. Most participants liked the vibrations which felt very similar to a known heartbeat [9]. 
There are already some commercial products which support the user in measuring the heart rate and in keeping it in defined borders. One example is the Garmin Forerunner $610^{3}$. Additionally to acoustic signals when the heart rate exceeds certain limits, it can also give tactile feedback in form of a vibration. Apps like Runkeeper ${ }^{4}$ can track a whole workout and also include heart rate recording, so the users can learn about their heart rate during physical activities.

Brewster et al. (2004) described with tactile icons (Tactons) a method to encode abstract messages with tactile feedback which can for example be used to display information without demanding other senses like sight and hearing [4]. Pielot et al. (2010) used tactons for location encoding and Asif et al (2010) for navigation tasks [3][7]. Both concluded that the tactile information display was equally or less distracting than visual or auditory information.

\section{Goals and Approach}

Our main goal of this paper is to prove whether users can perceive and interpret a pure tactile representation of the heart rate while walking and running outdoors. We further want to find out if this presentation enables users to adjust their physical activity to reach a previously defined target heart frequency, how accurate they are, and whether there are common deviations between perceived and target heart frequency, depending on the actual physical effort performed by the user. Additionally, we want to collect more qualitative data, such as emotions and suggestions from the users. We are interested in whether the tactile representation is disturbing, distracting, or annoying the user and whether it influences the walking or running rhythms. We also want to find out the subjective impression of how accurate the users reached the target heart rate.

Our approach to find answers to these questions was to develop a simple smart phone application for Android which presents the heart rate by vibration pulses of the smart phone. In this first study, we displayed each heartbeat by one vibration pulse, i.e. making the heart activity directly perceivable for the user, with the intention to provide immediate feedback when the heart frequency changes and to increase the users' experience by enabling them to immerse into the most natural representation of a physical load. Thus, we also avoid the more abstract presentation of heartbeats per minutes, which needs to be interpreted by the user.

\section{$4 \quad$ Field Study}

To investigate if the concept of a tactile pulse representing the heartbeat is applicable, we conducted a field study with a strong focus on qualitative feedback.

\footnotetext{
${ }^{3}$ http://sites.garmin.com/forerunner610/?lang=en

${ }^{4}$ http://runkeeper.com
} 


\subsection{Method and Setting}

In order to obtain results under realistic conditions, we decided to perform a field study instead of a lab study. After interviewing several medical experts, we provided users with target heart frequencies which should be reached during a physical exercise. To evaluate differences in the perception of different physical load levels, we defined three target heart rates. The three levels were defined as 80, 110, and $140 \mathrm{bpm}$ (beats per minute).

In the beginning of the study the participants received the apparatus and the experimenter explained the procedure of the study. The study consisted of three phases. They differed in the target heart rate which should be reached by the participant. The heart rate was chosen by the application randomly to avoid any learning bias in the study results. Each phase was divided into a learning part and a part with physical activity (e.g. running or walking). During the learning part, the target frequency is mediated to the user. The application plays the target frequency by vibration pulses in the same manner as described above, e.g. if the user should reach a target frequency of 110 beats per minute, the application presents 110 vibration pulses per minute. This allows the user to learn the frequency. We thought this was necessary, because we didn't expect the participants to have any prior experience in measuring heart rates even without beeing physical active. The participants were allowed to do anything during the learning part to get used to the feeling of the displayed target heart rate. The learning part was not limited in time and was ended by the participants themselves. Afterwards, the device switched to the running part. During this part, the measured heart rate of the participant was represented as tactile heartbeat. The participants were asked to try to adjust their walking or running speed so that their heart rate was just as high as the previously learned heart rate. There was no time restriction to reach the target. Once the participants thought they reached it, they had to press a button on the screen and try to keep this target heart rate for two minutes. After these two minutes, the application switched to the learning phase to present the next target frequency.

Questionnaire and Post Interview Before the study, the participants received a questionnaire and the session ended with a semi-structured interview with some guiding questions. In the questionnaire, we asked the participants for their age, sex, and background knowledge:

1. Do you know about the meaning of the heart rate?

2. Did you use a heart rate monitor before?

3. Do you know about your heart rates at specific loads?

For the open interview, we specified the following guiding questions:

1. How good or bad was the representation of your heart rate?

2. Did the application increase your awareness of your heart rate?

3. To reach the desired heart rate, did you use your feeling or the vibration presented by the app? 
4. Did the application distract you?

5. Did the application disturb your walking/running rhythm?

Location For each session, the experimenter and the participant met at a location chosen by the participant, often an area which is frequently used by runners. The participants were allowed to choose any place, which has no slopes and which provides minimum risk, even when the participant would be distracted by the application.

Participants We conducted the field study with ten participants: Eight of them male and two female. The age ranged from 21 to 41 .

Apparatus To measure the heart rate, we used a Zephyr $\mathrm{HxM}^{5}$ heart rate sensor. As smart phone we used the Samsung Galaxy Nexus ${ }^{6}$, running Android 4.0. You can see both in Fig. 1.
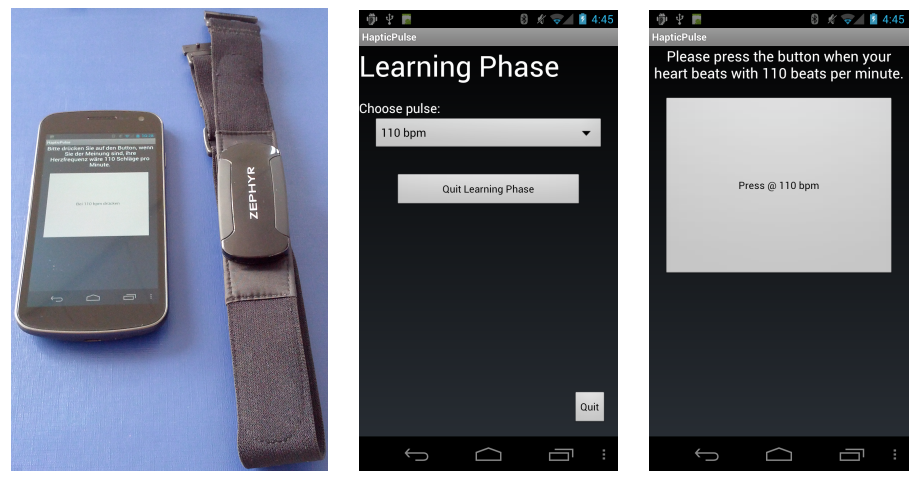

Fig. 1. HapticPulse Apparatus

In order to perform the study, we implemented an application for Android smart phones, which we call HapticPulse. The application consists of two screens (see Fig. 1): The first screen Learning Phase is intended to learn and feel a tactile representation of the heartbeat. The chosen frequency is selected randomly. The user also has the option to manually choose between the three different frequencies. After choosing a frequency it will immediately be presented through tactile pulses. The second screen is intended to instruct the user during the physical exercise (walking and running) and to allow the user to press a button when she or he thinks to have reached that goal. While this screen is shown, the heart

\footnotetext{
${ }^{5}$ http://www .zephyr-technology.com/consumer-hxm

${ }^{6}$ http://www. samsung.com/uk/consumer/mobile-devices/smartphones/android/ GT-I9250TSAXEU
} 
rate of the user is constantly represented as tactile pulse. Two minutes after the user indicated that the specified heart frequency is reached, the application switches to the first screen again. The application logs the heart rate reported by the heart rate sensor and any input from the user with time stamps. The participants were asked to carry the device in one of their hands.

\subsection{Quantitative results}

Seven participants knew about the meaning of the heart rate. Five had used some kind of heart rate display before, mostly watches. Four participants were even aware of their heart rates during specific activities. These participants were running frequently or had used a heart rate monitor in a fitness center. These data were collected through the questionnaire.

The $\log$ files of the application showed that the average duration of the learning parts was 179 seconds (SD 127 seconds) for $80 \mathrm{bpm}, 112$ seconds (SD 42 seconds) for $110 \mathrm{bpm}$, and 128 seconds (SD 66 seconds) for $140 \mathrm{bpm}$. The length of the learning parts seemed not to correlate with the overall performance. The average duration between switching to running mode and subjectively reaching the previous learned heart rate was 134 seconds (SD 89 seconds) for $80 \mathrm{bpm}, 46$ seconds (SD 47 seconds) for $110 \mathrm{bpm}$, and 70 seconds (SD 66 seconds) for 140 bpm. The average overall duration of the study, disregarding the explanation and interview, was 17 minutes (SD 4 minutes).

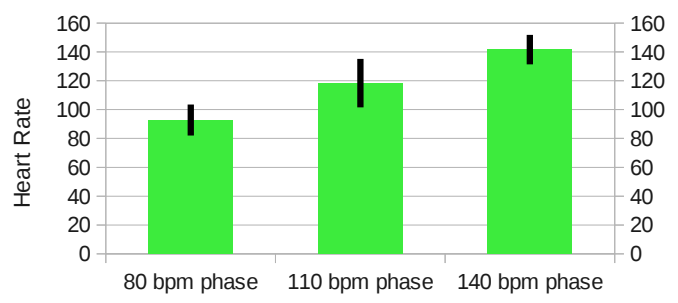

Fig. 2. Average heart rate and standard deviation when the button was pressed

Because we wanted the participants to press a button when they had reached the learned heart rate, it is interesting how accurate they were. As you can see in Fig. 2, the average heart rate for the $80 \mathrm{bpm}$ phase was $93 \mathrm{bpm}$ (SD $11 \mathrm{bpm}$ ). The average heart rate for the $110 \mathrm{bpm}$ phase was $118 \mathrm{bpm}$ (SD $17 \mathrm{bpm}$ ). In comparison with other participants, one participant had a very high value here. His deviation from the desired heart rate was five times higher compared to the other participants. If we eliminate this one measure, the average heart rate would be $114 \mathrm{bpm}$ (SD $7 \mathrm{bpm}$ ). The average heart rate for the $140 \mathrm{bpm}$ phase was $142 \mathrm{bpm}$ (SD $10 \mathrm{bpm})$. 


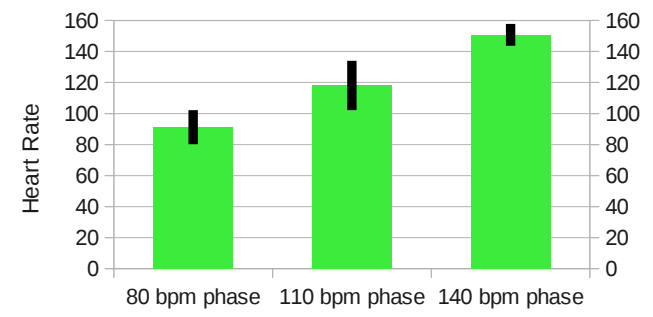

Fig. 3. Average heart rate and standard deviation during running phase

In Fig. 3 the average heart rates of the participants during the running phases are shown. It reveals that the participants had in average a higher heart rate than the target rate: $93 \mathrm{bpm}$ (SD $11 \mathrm{bpm}$ ) in the $80 \mathrm{bpm}$ phase, $122 \mathrm{bpm}$ (SD $16 \mathrm{bpm}$ ) in the $110 \mathrm{bpm}$ phase, and $152 \mathrm{bpm}(\mathrm{SD} 7 \mathrm{bpm}$ ) in the $140 \mathrm{bpm}$ phase.

\subsection{Qualitative Results}

In the interview, we used five guiding questions as mentioned before and gave the opportunity to give feedback.

How good or bad was the representation of your heart rate? The participants either answered positive or they did not answer because they had no idea of their heart rate and therefore were not able to classify the representation accuracy. One participant mentioned he would like a live representation and the delay caused him to rate the representation good at constant speeds but bad at changing speeds. It pointed out that he thought the application was rather slow when adapting to a new heart rate, but most of the delay he experienced came from his heart itself. Other participants reported a very direct representation of their heartbeat or that they were only able to feel the delay when standing still. One told us: "Very good. I was able to feel my heart rate very good. I think with an accuracy of 5 beats per minute.". In fact that participant had a deviation of about $6 \mathrm{bpm}$.

Did the application increase your awareness of your heart rate? Every participant stated that the application increased their awareness of their heart rate. Some mentioned it would be interesting to get this feedback over a longer period of time to learn about the reactions of the heart to different situations. The advantages of existing heart rate monitors for consumers was mentioned because they show a concrete numerical value. On the other hand one participant mentioned that he sometimes forgets about his heart rate monitor and likes this representation because it is constant and you can not forget about it. One described the feeling as "very intensive" because "you can feel very good how your body reacts". Another one would rather like an application telling him what to do, e.g. "run faster", instead of just showing the pure heart rate. 
To reach the desired heart rate, did you use your feeling or the vibration presented by the app? Most participants mainly used the application to reach the desired heart rate, especially when they had no idea about their heart rate during certain activities. It pointed out that more experience in running led to less usage of the application. One very active participant stated that "I used only my feelings to reach a specific heart rate. [...] I just used the application to be sure." while another inexperienced one said "The Application. Using my feelings wouldn't work since I have no idea about my heart rate when I am running.". Only one participant said he had big problems learning and remembering the heart rates in the learning phases. He also stated that he forgot about the vibration while running.

Did the application distract you? Four participants said they were not distracted by the application at all. One even stated that he liked the permanent representation instead of looking at a heart rate monitor screen. Three participants felt very distracted, because they had to concentrate on the application. They were not sure if that would be the same outside a study environment. Others were distracted because they had to hold the device in their hand all the time but not by the vibrations themselves. One participant mentioned that using the device in combination with music may be difficult because you hear and feel different rhythms.

Did the application disturb your walking/running rhythm? Four participants did not feel disturbed by the vibration of the application. One felt disturbed all the time. The other ones mentioned exactly one phase in which their heart rate and their running rhythm interfered, so they were not able to walk normally because they wanted to walk in the same speed as their heart beat.

Additional feedback Most of the participants did not like to hold a smart phone in their hands all the time. One suggested to use a vibrating ball instead which is easier to hold. Another one suggested to integrate the vibration into the chest belt of the heart rate sensor. The idea itself to display the heart rate with vibration was well accepted and described as comfortable. The vibration itself was not felt in the same way by all participants. Sometimes it was mentioned that the vibration was easy to feel ("It's surprising how good you can feel it when running.") but also that it was hard to feel ("The vibration should be stronger. It's hard to feel while running."). One participant even put the device on his ear and said "it's easier to hear than to feel". It was also suggested not to communicate every heartbeat but just every second one, because at higher heart rates the vibrations are too frequent. In addition it was recommended that the device be easy to switch on and off. Some participants thought that using the device for a longer period of time would result in a good feeling for the displayed frequency and therefore for the own heart rate. It was seen positively 
that the application gives permanent feedback about the activity level. Generally, the application was well accepted and commented as "cool", "exciting", and "interesting". Some participants missed features of regular heart rate displays like warnings at certain heart rates, but liked the permanent feedback.

\subsection{Discussion}

The interviews have shown that our participants generally like the idea of a tactile heart rate display. They were often able to tell when their heart rate deviated from the learned one by more than $10 \mathrm{bpm}$. The high deviation at 80 bpm for some participants is not surprising, because some reported that they were hardly able to reach such a low heart rate during the study or just did not want to wait until their heart rate dropped so low. In all cases but one the measured heart rate was a bit higher than the learned one. Most participants had a lower heart rate when they ended the learning part, so they may have wanted to raise it faster and started to walk or run faster than necessary. If such a tendency can be confirmed in future evaluations, a correction factor to the tactile pulse could be applied so the users would more likely reach the desired heart rate.

It is remarkable that all participants stated that the haptic pulse strengthened the awareness of their heart rate. This is very interesting when thinking about special needs of certain user groups. People with a heart disease, for example, may use such a haptic pulse device to learn more about their heart, not just during physical exercises, but also in their everyday live. Some participants even learned something about the behaviour of their heart during our short study, so a bigger learning effect may appear when using a haptic pulse device for a longer time period. To address the need for a warning at certain heart rates, some methods to simultaneously display the heart rate and these warnings have to be evaluated. Due to the fact that some participants felt disturbed at specific pulse rates it may be useful to make the device easy to turn off when it is used for a short time, like a heart rate watch.

A vibrating smart phone held in the hands seems to be in no way sufficient, especially when the device should be used over a longer period of time. The device is too loud in silent environments and the vibration is too weak when the device is not held in the hands during exercises. A device which can be safely attached to a sensitive part of the body and generates vibration impulses more or less intense with respect to the activity of the wearers may help to improve their experience.

\section{Conclusions and Future Work}

In this paper we evaluated a tactile representation of the heart rate during physical activities. In a user study with ten participants we collected qualitative feedback which can be used to built improved devices and enable a good user experience when a permanent heart rate display is needed or useful. Since many 
participants were also interested in a long term usage of the device, future research and development of wearable sensors and actors was encouraged. Because of the great improvement of the awareness of the heart rate, tests with people who actually need to learn about the behaviour of their heart rate like patients with a heart disease would be interesting. Regarding this learning effect it is also interesting whether the device can be removed and after what time span this is possible without loosing the benefits. The device could for example reduce the feedback over time, so that at first it displays the heart rate all the time and later only in certain situations or on demand of the user. Incorporating the technique into other health related applications, where the pure display and recording of the heart rate is not enough, may be useful. So they can benefit from the possible

lower distraction and the learning effect. After all, we see a great potential in displaying the heart rate in a tactile way. In the future this concept may also be applied to other vital parameters like the breathing frequency to raise the awareness for reactions of the body to certain situations.

\section{References}

1. J. Achten and A. Jeukendrup. Heart rate monitoring. Sports Med, 33(7):517-538, 2003.

2. C. Albinet, G. Boucard, C. Bouquet, and M. Audiffren. Increased heart rate variability and executive performance after aerobic training in the elderly. European journal of applied physiology, 109(4):617-624, 2010.

3. A. Asif and S. Boll. Where to turn my car?: comparison of a tactile display and a conventional car navigation system under high load condition. In Proceedings of the 2nd International Conference on Automotive User Interfaces and Interactive Vehicular Applications, pages 64-71. ACM, 2010.

4. S. Brewster and L. Brown. Tactons: structured tactile messages for non-visual information display. In Proceedings of the fifth conference on Australasian user interface-Volume 28, pages 15-23. Australian Computer Society, Inc., 2004.

5. F. Gamelin, S. Berthoin, H. Sayah, C. Libersa, and L. Bosquet. Effect of training and detraining on heart rate variability in healthy young men. International journal of sports medicine, 28(7):564-570, 2007.

6. G. Lotan and C. Croft. impulse. In CHI'O7 extended abstracts on Human factors in computing systems, pages 1983-1988. ACM, 2007.

7. M. Pielot, O. Krull, and S. Boll. Where is my team: supporting situation awareness with tactile displays. In Proceedings of the 28th international conference on Human factors in computing systems, pages 1705-1714. ACM, 2010.

8. J. Thayer, F. Åhs, M. Fredrikson, J. Sollers III, and T. Wager. A meta-analysis of heart rate variability and neuroimaging studies: Implications for heart rate variability as a marker of stress and health. Neuroscience 85 Biobehavioral Reviews, 2011.

9. J. Werner, R. Wettach, and E. Hornecker. United-pulse: feeling your partner's pulse. In Proceedings of the 10th international conference on Human computer interaction with mobile devices and services, pages 535-538. ACM, 2008.

10. U. Wisløff, A. Støylen, J. Loennechen, M. Bruvold, Ø. Rognmo, P. Haram, A. Tjønna, J. Helgerud, S. Slørdahl, S. Lee, et al. Superior cardiovascular effect of aerobic interval training versus moderate continuous training in heart failure patients. Circulation, 115(24):3086-3094, 2007. 